Commun. Korean Math. Soc. 29 (2014), No. 1, pp. 163-172

http://dx.doi.org/10.4134/CKMS.2014.29.1.163

\title{
ATTRACTORS AND LYAPUNOV FUNCTION FOR CLOSED RELATIONS
}

\author{
Gui Seok Kim, Kyung Bok Lee, and Jong Suh Park
}

\begin{abstract}
In this paper, we will study the characterizations of attractors and Lyapunov function which were studied in flows and homeomorphisms will also be satisfied in compact closed relation dynamics. In particular, we will find the necessary and sufficient conditions of the existence of the strict Lyapunov function.
\end{abstract}

\section{Introduction and preliminaries}

The main purpose of this paper is to prove following two theorems.

Theorem 2.10. Let $A$ be a $N f$-positively invariant closed subset of $X$. Then there exists a Lyapunov function $h: X \rightarrow[0,1]$ for $f$ such that $A=h^{-1}(0)$.

Theorem 2.11. Let $f$ be a compact continuous relation on a locally compact space $X$ and $A$ be a nonempty compact invariant subset of $X$. Assume that there exists a strict Lyapunov function $h: X \rightarrow[0, \infty)$ for $f$ satisfying $h^{-1}(0)=A$. Then $A$ is an attractor for $f$.

To begin with, we introduce the necessary definitions and results without proof.

Let $\left(X_{1}, d_{1}\right),\left(X_{2}, d_{2}\right)$ be arbitrary metric spaces. A relation $f: X_{1} \rightarrow X_{2}$ is considered as a map from $X_{1}$ to the power set of $X_{2}$, that is, each $x \in X_{1}$ corresponds to a subset $f(x)$ of $X_{2}$, or a subset of $X_{1} \times X_{2}$ so that $y \in f(x)$ means $(x, y) \in f$. We define the domain of $f$ by

$$
\operatorname{Dom}(f)=\left\{x \in X_{1} \mid f(x) \neq \emptyset\right\} .
$$

i.e., $x \in \operatorname{Dom}(f)$ if and only if there exists $y \in X_{2}$ such that $(x, y) \in f$.

For relations $f: X_{1} \rightarrow X_{2}$ and $g: X_{2} \rightarrow X_{3}$ we define the inverse $f^{-1}$ : $X_{2} \rightarrow X_{1}$, and the composition $g \circ f: X_{1} \rightarrow X_{3}$ by

$$
x \in f^{-1}(y) \Longleftrightarrow y \in f(x),
$$

Received July 31, 2013.

2010 Mathematics Subject Classification. 37B40.

Key words and phrases. closed relation, attractors, Lyapunov function. 


$$
\text { i.e., } \begin{aligned}
f^{-1} & =\{(y, x) \mid(x, y) \in f\} \text {, and } \\
y & \in(g \circ f)(x) \Longleftrightarrow z \in f(x) \text { and } y \in g(z) \text { for some } z \in X_{2} .
\end{aligned}
$$

The usual composition properties of associativity, identity, and inversion generalize to the relation, e.g., $1_{X_{2}} \circ f=f=f \circ 1_{X_{1}}$ and $(g \circ f)^{-1}=f^{-1} \circ g^{-1}$. There are additional algebraic properties as well. For example, composition distributes over the union:

$$
\left(\cup_{m} g_{m}\right) \circ\left(\cup_{n} f_{n}\right)=\cup_{m, n}\left(g_{m} \circ f_{n}\right) .
$$

For $f: X \rightarrow X$ we define $f^{n}$ to be the $n$-fold composition of $f(n=0,1,2, \cdots$ with $f^{0}=1_{X}$ and $f^{1}=f$ by definition). $f^{-n}$ is defined to be $\left(f^{-1}\right)^{n}$ (which equals $\left.\left(f^{n}\right)^{-1}\right)$. For a relation $f: X_{1} \rightarrow X_{2}$ and a subset $A$ of $X_{1}$ the image $f(A) \subset X_{2}$ is defined by

$$
f(A)=\{y \mid(x, y) \in f \text { for some } x \in A\}=\cup\{f(x) \mid x \in A\} .
$$

$f: X_{1} \rightarrow X_{2}$ is called a closed relation if it is a closed subset of $X_{1} \times X_{2}$.

$f: X_{1} \rightarrow X_{2}$ is called a compact relation if $f(x)$ is a compact subset of $X_{2}$ for any $x \in X_{1}$.

Definition $1.1([1])$. A relation $f: X_{1} \rightarrow X_{2}$ is said to be upper semicontinuous at $x \in X_{1}$ if for every $\epsilon>0$ there exists a $\delta>0$ such that

$$
d_{1}(x, y)<\delta \text { implies } f(y) \subset B(f(x), \epsilon) .
$$

A relation $f: X_{1} \rightarrow X_{2}$ is said to be lower semicontinuous at $x \in X_{1}$ if for every $\epsilon>0$ there exists a $\delta>0$ such that

$$
d_{1}(x, y)<\delta \text { implies } f(x) \subset B(f(y), \epsilon) .
$$

A relation $f$ is said to be continuous at $x$ if $f$ is both upper semicontinuous at $x$ and lower semicontinuous at $x$.

Proposition 1.1. Let $f$ be a relation on $X$ and $x \in X$. Then the following are true: (1) $f$ is upper semicontinuous at $x$ if and only if for sequences $\left(x_{n}\right)$, $\left(y_{n}\right)$ in $X$ with $x_{n} \rightarrow x, y_{n} \rightarrow y$, and $y_{n} \in f\left(x_{n}\right), y \in f(x)$. (2) $f$ is lower semicontinuous at $x$ if and only if for every sequence $\left(x_{n}\right),\left(y_{n}\right)$ in $X$ with $x_{n} \rightarrow x, y_{n} \rightarrow y$, and $y_{n} \in f\left(x_{n}\right), d\left(y_{n}, f\left(x_{n}\right)\right)$ converges to 0 as $n \rightarrow \infty$.

Lemma 1.2. Let $f: X_{1} \rightarrow X_{2}$ be a compact relation. (1) $f$ is upper semicontinuous at $x$ if and only if for every neighborhood $U$ of $f(x)$, there exists a neighborhood $V$ of $x$ such that $y \in V$ implies $f(y) \subset U$. (2) $f$ is lower semicontinuous at $x$ if and only if for every open set $U$ with $U \cap f(x) \neq \emptyset$, there exists a neighborhood $V$ of $x$ such that $y \in V$ implies $f(y) \cap U \neq \emptyset$.

Theorem 1.3. Let $f: X_{1} \rightarrow X_{2}$ be compact lower semicontinuous. Then $f(\bar{A}) \subset \overline{f(A)}$ for any subset $A$ of $X_{1}$.

Proposition 1.4. Let $f: X_{1} \rightarrow X_{2}$ be compact upper semicontinuous and let $X_{2}$ be a locally compact space. Then for every compact subset $K$ of $X_{1}, f(K)$ is a compact subset of $X_{2}$. 
Proposition 1.5. Let $f: X_{1} \rightarrow X_{2}$ be compact continuous and let $X_{2}$ be locally compact. For any compact subset $C$ of $X_{1}$ and neighborhood $U$ of $f(C)$ there exists a neighborhood $V$ of $C$ such that $f(V) \subset U$.

Corollary 1.6. Let $(X, d)$ be a locally compact space and $f: X \rightarrow X$ be compact continuous. Then $n$-fold composition of $f, f^{n}: X \rightarrow X$, is compact continuous.

\section{Attractors and Lyapunov function}

Many mathematicians have been researched on the attractor and Lyapunov function for flows and homeomorphisms ([2], [3]). This section is devoted to the study of notions of attractor and the characterization of some properties of them in terms of the so called Lyapunov function.

Let $f$ be a closed continuous relation on $X$.

For every subset $A$ of $X$, the $\omega$-limit set $\omega(A)$ of $A$ is denoted to be the set:

$$
\omega(A)=\cap_{n \geq 0} \overline{\cup_{k \geq n} f^{k}(A)} .
$$

Now, let us explore the properties of $\omega$-limit set.

Proposition 2.1. Let $A$ be a subset of $X$. Then $x \in \omega(A)$ if and only if there exist sequences $\left(a_{i}\right)$ in $A,\left(n_{i}\right)$ in $\mathbb{N}$, and $\left(x_{i}\right)$ in $X$ such that $n_{i} \rightarrow \infty$, $x_{i} \in f^{n_{i}}\left(a_{i}\right)$, and $x_{i} \rightarrow x$.

Proof. Let $x \in \omega(A)$. For every $i \in \mathbb{N}$, since $x \in \overline{\cup_{n \geq i} f^{n}(A)}$,

$$
B\left(x, \frac{1}{i}\right) \cap \cup_{n \geq i} f^{n}(A) \neq \emptyset .
$$

Let $x_{i} \in B\left(x, \frac{1}{i}\right) \cap \cup_{n \geq i} f^{n}(A)$. Then there exist $n_{i} \geq i, a_{i} \in A$ such that $x_{i} \in f^{n_{i}}\left(a_{i}\right)$. It is clear that $n_{i} \rightarrow \infty$ and $x_{i} \rightarrow x$.

Suppose that there exist sequences $\left(a_{i}\right)$ in $A,\left(n_{i}\right)$ in $\mathbb{N}$, and $\left(x_{i}\right)$ in $X$ such that $n_{i} \rightarrow \infty, x_{i} \in f^{n_{i}}\left(a_{i}\right)$, and $x_{i} \rightarrow x$. Then for every $n \geq 0$ and neighborhood $U$ of $x$, there exist $x_{i} \in U$ and $n_{i} \geq n$. Since $x_{i} \in f^{n_{i}}\left(a_{i}\right) \subset$ $f^{n_{i}}(A) \subset \cup_{k \geq n} f^{k}(A), U \cap \cup_{k \geq n} f^{k}(A) \neq \emptyset$. Thus $x \in \omega(A)=\cap_{n \geq 0} \overline{\cup_{k \geq n} f^{k}(A)}$.

Definition 2.1. A subset $A$ of $X$ is said to be positively invariant if $f(A) \subset A$. A subset $A$ of $X$ is said to be invariant if $f(A)=A$ or equivalently if $A$ is positively invariant and $f^{-1}(x) \cap A \neq \emptyset$ for all $x \in A$.

Proposition 2.2. For every subset $A$ of compact metric space $X, \omega(A)$ is closed and invariant.

Proof. By definition of $\omega$ limit set, $\omega(A)$ is closed.

$$
\begin{aligned}
f(\omega(A))=f\left(\cap_{n \geq 0} \overline{\cup_{k \geq n} f^{k}(A)}\right) & \subset \cap_{n \geq 0} f\left(\overline{\cup_{k \geq n} f^{k}(A)}\right) \\
& \subset \cap_{n \geq 0} \overline{f\left(\cup_{k \geq n} f^{k}(A)\right)} \\
& =\cap_{n \geq 0} \overline{\cup_{k \geq n} f^{k+1}(A)}
\end{aligned}
$$




$$
\begin{aligned}
& \subset \cap_{n \geq 0} \overline{\cup_{k \geq n} f^{k}(A)} \\
& =\omega(A) .
\end{aligned}
$$

Let $x \in \omega(A)$. By Proposition 2.1, there exist sequences $\left(a_{i}\right)$ in $A,\left(n_{i}\right)$ in $\mathbb{N}$, and $\left(x_{i}\right)$ in $X$ such that $n_{i} \rightarrow \infty, x_{i} \in f^{n_{i}-1}\left(a_{i}\right)$, and $x_{i} \rightarrow x$. Since $x_{i} \in f^{n_{i}}\left(a_{i}\right)=f\left(f^{n_{i}-1}\left(a_{i}\right)\right)$, we can find $y_{i} \in f^{n_{i}-1}\left(a_{i}\right)$ with $x_{i} \in f\left(y_{i}\right)$. By compactness of $X$, the sequence $\left(y_{i}\right)$ has a convergent subsequence. Let $y_{i} \rightarrow y$. Since $n_{i}-1 \rightarrow \infty, y \in \omega(A)$. By Proposition 2.1, $x \in f(y) \subset f(\omega(A))$. Thus $f(\omega(A))=\omega(A)$.

Proposition 2.3. If $A \subset X$ is positively invariant, then $\bar{A}$ is positively invariant.

Proof. Since $f(\bar{A}) \subset \overline{f(A)} \subset \bar{A}, \bar{A}$ is positively invariant.

Proposition 2.4. If $A \subset X$ is $f$-positively invariant, then $A$ is $\bar{f}$-positively invariant.

Proof. Let $y \in \bar{f}(A)$, then there exists $x \in A$ such that $(x, y) \in \bar{f}$. There exists a sequence $\left(\left(x_{n}, y_{n}\right)\right)$ in $f$ such that $\left(x_{n}, y_{n}\right) \rightarrow(x, y)$. Since $x_{n} \rightarrow x, y_{n} \rightarrow y$, and $y_{n} \in f\left(x_{n}\right)$ for all $n$, we have $y \in f(x) \subset f(A) \subset A$. Thus $\bar{f}(A) \subset A$.

We start with the definitions of attractor and attractor block.

Definition $2.2([3])$. A subset $A$ of $X$ is said to attractor for $f$ if there exists a neighborhood $U$ of $A$ such that $\omega(U)=A$.

By Proposition 2.2, attractor is a closed invariant set.

Definition $2.3([3])$. A subset $B$ of $X$ is said to attractor block for $f$ if $f(\bar{B}) \subset$ $\operatorname{Int}(B)$.

Theorem 2.5. Attractor block $B$ is a neighborhood $\omega(B)$. Thus $\omega(B)$ is an attractor.

Proof. Since $\bar{B}$ is compact, $f(\bar{B})$ is compact. Thus $\overline{f(\bar{B})}=f(\bar{B})$.

$$
\omega(B)=\cap_{n \geq 0} \overline{f^{n}(B)} \subset \overline{f(B)} \subset \overline{f(\bar{B})}=f(\bar{B}) \subset \operatorname{Int}(B) .
$$

Thus $B$ is a neighborhood $\omega(B)$.

Theorem 2.6. If $A$ is an attractor for $f$ and $V$ is a neighborhood of $A$, then there exists a closed attractor block $B$ for $f$ such that $B \subset V$ and $\omega(B)=A$.

Proof. Since $A$ is an attractor, there exists a neighborhood $U$ of $A$ such that $\omega(U)=A$. For a neighborhood $U \cap A$ of $A$, there exists a closed neighborhood $B$ of $A$ such that $B \subset U \cap V$. Since $A \subset B \subset U$, we have

$$
A=\omega(A) \subset \omega(B) \subset \omega(U)=A .
$$

Thus $\omega(B)=A$. 
Suppose that $A$ is an attractor, that $B$ is an attractor block, and that $A=\omega(B)$. Then $A$ will be called the attractor associated with $B$, while $B$ will be called an attractor block associated with $A$. Theorem 2.5 states that an attractor block always has an associated attractor. Theorem 2.6 states that an attractor always has an associated attractor block in any neighborhood.

For a relation $f$ on $X$, we define the relations $O f, N f$ on $X$ by

$$
O f=\cup_{n \in \mathbb{N}} f^{n}, N f=\overline{O f} .
$$

Proposition 2.7. If $A \subset X$ is an $f$-positively invariant closed set, then $A$ is $\mathrm{Nf}$-positively invariant.

Proof. Since $f(A) \subset A$, we have

$$
A \supset f(A) \supset f^{2}(A) \supset \cdots .
$$

Thus $O f(A)=\cup_{n \in \mathbb{N}} f^{n}(A)=f(A) \subset A$. By Proposition 2.4, we obtain $N f(A)=\overline{\cup_{n \in \mathbb{N}} f^{n}(A)} \subset A$.

Proposition 2.8. For a closed subset $A$ of $X$, the following conditions are equivalent: (1) $N f(A) \subset A$ (2) For every neighborhood $U$ of $A$ there exists a neighborhood $V \subset U$ of $A$ such that $O f(x) \subset U$ for all $x \in V$. (3) For every neighborhood $U$ of $A$ there exists a neighborhood $V \subset U$ of $A$ such that $f(U) \subset V$.

Proof. (1) $\Rightarrow(3)$. Since $N f$ is a closed relation on $X$, there exists $\delta>0$ such that $B[A, \delta] \subset U$ and $N f(B[A, \delta]) \subset U$. Let $V=B(A, \delta) \cup O f(B(A, \delta))$. Since $B(A, \delta) \subset V, V$ is a neighborhood of $A$. Clearly, $f(V) \subset V$. We have

$$
V \subset B[A, \delta] \cup N f(B[A, \delta]) \subset U .
$$

$(3) \Rightarrow(2)$. This is obvious.

$(2) \Rightarrow(1)$. Let $x \in A$ and $y \in N f(x)$. Given any $\epsilon>0$, since $B(A, \epsilon)$ is a neighborhood of $A$, there exists a neighborhood $U \subset B(A, \epsilon)$ of $A$ such that $O f(x) \subset B(A, \epsilon)$ for all $x \in U$. There exists $\delta$ with $0<\delta<\epsilon$ such that $B(A, \delta) \subset U$. Since

$$
(x, y) \in N f=\overline{O f}
$$

we have

$$
(B(x, \delta) \times B(y, \delta)) \cap O f \neq \emptyset
$$

and so $(B(x, \delta) \times B(y, \delta)) \cap f^{n} \neq \emptyset$ for some $n \geq 1$. Let $(p, q) \in(B(x, \delta) \times$ $B(y, \delta)) \cap f^{n}$. Then $d(x, p)<\delta, d(y, q)<\delta$ and $q \in f^{n}(p)$. Since $d(p, A) \leq$ $d(p, x)<\delta, p \in B(A, \delta) \subset U$. Thus $q \in f^{n}(p) \subset B(A, \epsilon)$. Then $d(y, A) \leq$ $d(y, q)+d(q, A)<\delta+\epsilon<2 \epsilon$. Hence $d(y, A)=0$ and so $y \in A$. Therefore $N f(A) \subset A$.

Proposition 2.9. Let $f$ be a transitive relation on $X$ and $A$ be a closed positively invariant subset of $X$. For every neighborhood $U$ of $A$, there exist positively invariant open set $V_{1}$ and positively invariant closed set $V_{2}$ such that

$$
A \subset V_{1} \subset V_{2} \subset U \text {. }
$$


Proof. Since $f$ is transitive, we have $N f=O f=f$. Since $N f(A)=f(A) \subset A$, by the proof of $(1) \Rightarrow(3)$ in Proposition 2.8, $V_{2}=B[A, \delta] \cup f(B[A, \delta]) \subset U$ is a positively invariant closed set. Let $V_{1}=\left\{x \in \operatorname{Int}\left(V_{2}\right) \mid f(x) \subset \operatorname{Int}\left(V_{2}\right)\right\}$. Since $f$ is transitive, $V_{1}$ is a positively invariant open set. Since $A$ is positively invariant and $B(A, \delta) \subset \operatorname{Int}\left(V_{2}\right)$, we have $A \subset V_{1}$.

We now introduce the fundamental definitions of Lyapunov function and strict Lyapunov function.

Definition 2.4. Let $f$ be a compact continuous relation on $X$. We say that $h$ is a Lyapunov function for $f$ if $h: X \rightarrow[0, \infty)$ is continuous, $A=h^{-1}(0)$ is nonempty invariant and $h(y) \leq h(x)$ for all $x \in X, y \in f(x)$.

Remark 2.1. In general, the Lyapunov function $h$ need not have the property $m<n$ implies $\max h\left(f^{n}(x)\right)<\min h\left(f^{m}(x)\right)$ for all $x \notin A$.

For example, let $X=[0,1], h: X \rightarrow[0, \infty)$ be the identity function and let $f=\left\{(x, y) \in X \times X \mid \frac{1}{3} x \leq y \leq \frac{2}{3} x\right\}$. Then $h$ is a Lyapunov function, but $h$ does not satisfy the above condition. So we introduce the following.

Definition 2.5. A Lyapunov function $h$ is called a strict Lyapunov function if there is a neighborhood $W$ of $A$ such that $h^{-1}([0,1]) \subset W$ and $m<n$ implies $\max h\left(f^{n}(x)\right)<\min h\left(f^{m}(x)\right)$ for all $x \in W-A$. In particular, if $h$ is a strict Lyapunov function, then $h(y)<h(x)$ for all $x \notin A, y \in f(x)$.

We now give an important characterization of Lyapunov function.

Theorem 2.10. Let $A$ be a $N f$-positively invariant closed subset of $X$. Then there exists a Lyapunov function $h: X \rightarrow[0,1]$ for $f$ such that $A=h^{-1}(0)$.

Proof. Let $R$ be the set of all rationals $r$ of form $\frac{k}{2^{n}}, 0<\frac{k}{2^{n}} \leq 1$. We first show that with $r \in R$, we can associate an $N f$-positively invariant open subset $U(r)$ of $X$ such that

(1) $A \subset U(r)$,

(2) $r_{1}<r_{2}$ implies $\overline{U\left(r_{1}\right)} \subset U\left(r_{2}\right)$.

We proceed by induction on the exponent of the dyadic fractions, letting

$$
D_{m}=\left\{U\left(\frac{k}{2^{m}}\right) \mid k=1,2, \ldots, 2^{m}\right\} .
$$

$D_{1}$ consists of $U(1)=X$ and $U\left(\frac{1}{2}\right)=$ some $N f$-positively invariant open set satisfying

$$
A \subset U\left(\frac{1}{2}\right) \subset \overline{U\left(\frac{1}{2}\right)} \subset U(1),
$$

which exists by Proposition 2.9. Assume $D_{m-1}$ constructed, and note that only $U\left(\frac{k}{2^{m}}\right)$ for odd $k$ requires definition; for each odd $k$, we have from $D_{m-1}$ that

$$
\overline{U\left(\frac{k-1}{2^{m}}\right)} \subset U\left(\frac{k+1}{2^{m}}\right),
$$


so we define $U\left(\frac{k}{2^{m}}\right)$ to be an $N f$-positively invariant open set $U$ satisfying

$$
\overline{U\left(\frac{k-1}{2^{m}}\right)} \subset U \subset \bar{U} \subset U\left(\frac{k+1}{2^{m}}\right) .
$$

This completes the inductive step.

Let $D=\cup_{m \in \mathbb{N}} D_{m}$ and let

$$
h(x)=\inf \{r \mid x \in U(r)\} .
$$

Clearly, $0 \leq h(x) \leq 1$. Furthermore $h(A)=0$, since $A \subset U(r)$ for all $r$. Because $U(r)$ is positively invariant, $x \in U(r)$ implies $f(x) \subset U(r)$ and so for $y \in f(x)$ we have

$$
\{r \mid x \in U(r)\} \subset\{r \mid y \in U(r)\} .
$$

Thus $h(y)$, the sup of the larger set, is at least $h(x)$.

It remains to prove continuity. Let $h\left(x_{0}\right)=r_{0}$, and choose any $W=\left(r_{0}-\right.$ $\left.\epsilon, r_{0}+\epsilon\right)$. If $r_{0} \neq 0,1$, choose also $r_{1}, r_{2}$ such that

$$
r_{0}-\epsilon<r_{1}<r_{0}<r_{2}<r_{0}+\epsilon,
$$

then $U=U\left(r_{2}\right)-\overline{U\left(r_{1}\right)}$ is a neighborhood of $x_{0}$ and $h(U) \subset W$, since $x \in U\left(r_{2}\right)$ implies $h(x) \leq r_{2}$, and $x \notin \overline{U\left(r_{1}\right)}$ implies $h(x) \geq r_{1}$. If $r_{0}=0$ (or 1 ), then the neighborhood $U\left(r_{2}\right)$ (or $\left.X-\overline{U\left(r_{1}\right)}\right)$ of $x_{0}$ alone suffices. This completes the proof.

We are now in a position to prove the following important result.

Theorem 2.11. Let $f$ be a compact continuous relation on a locally compact space $X$ and $A$ be a nonempty compact invariant subset of $X$. Assume that there exists a strict Lyapunov function $h: X \rightarrow[0, \infty)$ for $f$ satisfying $h^{-1}(0)=A$. Then $A$ is an attractor for $f$.

Proof. Since $X$ is locally compact, there is a compact neighborhood $K$ of $A$ such that $k \subset W$. For any $\epsilon>0$, let $K_{\epsilon}=\{x \in K \mid h(x) \leq \epsilon\}$. Then $K_{\epsilon}$ is a compact subset of $K$. We claim that for every neighborhood $U$ of $A$ there exists a constant $\delta<0$ such that $0<\epsilon \leq \delta$ implies $K_{\epsilon} \subset U$.

Suppose that the above claim is not true. Then there is a neighborhood $U$ of $A$ with the property that, for every $\delta>0$, there is a positive constant $\epsilon$ with $0<\epsilon \leq \delta$ such that $K_{\epsilon} \not \subset U$. For every $n \in \mathbb{N}$, there is a positive constant $\epsilon_{n}$ with $0<\epsilon_{n} \leq \frac{1}{n}$ such that $K_{\epsilon_{n}} \not \subset U$. Then there is $x_{n} \in K_{\epsilon_{n}}-U \subset K-U$. Since $K-U$ is compact, $\left(x_{n}\right)$ has a convergent subsequence. Let $x_{n} \rightarrow p \in$ $K-U$. Since $p \notin A, h(p)>0$. Since $h\left(x_{n}\right) \rightarrow h(p)$ and $0 \leq h\left(x_{n}\right) \leq \epsilon_{n} \leq \frac{1}{n}$, $h(p)=0$. This is a contradiction.

For every $x \in A$,

$$
f(x) \subset f(A)=A \subset \operatorname{Int}(K) .
$$

Since $f(x)$ is compact, there is $\epsilon_{x}>0$ such that $B\left(f(x), \epsilon_{x}\right) \subset \operatorname{Int}(K)$. Since $f$ is continuous at $x$, there exists $\delta_{x}>0$ such that $B\left(x, \delta_{x}\right) \subset \operatorname{Int}(K)$ and $d(x, y)<\delta_{x}$ implies $f(y) \subset B\left(f(x), \epsilon_{x}\right) .\left\{B\left(x, \delta_{x}\right) \mid x \in A\right\}$ is an open 
cover of $A$. Since $A$ is compact, there are finitely many elements $x_{1}, x_{2}, \ldots, x_{n}$ in $A$ such that

$$
A \subset \cup_{i=1}^{n} B\left(x_{i}, \delta_{x_{i}}\right) .
$$

Let $U=\cup_{i=1}^{n} B\left(x_{i}, \delta_{x_{i}}\right)$. Then $U$ is a neighborhood of $A$ and $U \subset \operatorname{Int}(K)$. For every $y \in U$, there is $i$ such that $y \in B\left(x_{i}, \delta_{x_{i}}\right)$. Since $d\left(x_{i}, y\right)<\delta_{x_{i}}$,

$$
f(y) \subset B\left(f\left(x_{i}\right), \epsilon_{x_{i}}\right) \subset \operatorname{Int}(K) .
$$

Thus $f(U) \subset \operatorname{Int}(K)$. There is $\delta>0$ such that $0<\epsilon \leq \delta$ implies $K_{\epsilon} \subset U$. We claim that $f\left(K_{\epsilon}\right) \subset \operatorname{Int}\left(K_{\epsilon}\right)$. Let $x \in K_{\epsilon}$. There are two cases depending upon whether $x \in A$ or not.

For the first case $x \in A$, we have $f(x) \subset f(A)=A$. Since $h^{-1}(0)=A$, we have $f(x) \subset \operatorname{Int}\left(K_{\epsilon}\right)$.

For the remaining case $x \notin A$, since $h(y)<f(x) \leq \epsilon$ for all $y \in f(x)$, we have $f(x) \subset \operatorname{Int}\left(K_{\epsilon}\right)$. Thus $f\left(K_{\epsilon}\right) \subset \operatorname{Int}\left(K_{\epsilon}\right)$ and so $K_{\epsilon}$ is an attractor block.

Since $A=f^{n}(A) \subset f^{n}\left(K_{\epsilon}\right)$ for all $n \geq 0$, we obtain

$$
A \subset \cap_{n=0}^{\infty} f^{n}\left(K_{\epsilon}\right) \subset \cap_{n=0}^{\infty} \overline{f^{n}\left(K_{\epsilon}\right)} .
$$

Now we will prove that $\cap_{n=0}^{\infty} \overline{f^{n}\left(K_{\epsilon}\right)} \subset A$. Since $K_{\epsilon}$ is compact, $f^{n}\left(K_{\epsilon}\right)$ is compact by Proposition 2.4. So $\overline{f^{n}\left(K_{\epsilon}\right)}=f^{n}\left(K_{\epsilon}\right)$. Thus it suffices to show that $\cap_{n=0}^{\infty} f^{n}\left(K_{\epsilon}\right) \subset A$.

Let $x \in K_{\epsilon}-A$ and $y_{n} \in f^{n}(x)$ for every $n$. We claim that

$$
h\left(y_{n}\right) \rightarrow 0 \text { as } n \rightarrow \infty .
$$

Suppose that above statement is not true. Then there exists $\epsilon>0$ such that for every $n \in \mathbb{N}$, there is a natural number $N>n$ such that $h\left(y_{N}\right) \geq \epsilon$. Since

$$
K_{\epsilon} \supset f\left(K_{\epsilon}\right) \supset f^{2}\left(K_{\epsilon}\right) \supset \cdots,
$$

we have

$$
y_{n_{i}} \in f^{n_{i}}(x) \subset f^{n_{i}}\left(K_{\epsilon}\right) \subset K_{\epsilon} .
$$

Since $K_{\epsilon}$ is compact, $\left(y_{n_{i}}\right)$ has a convergent subsequence. Let $y_{n_{i}} \rightarrow p \in K_{\epsilon}$. Then $h\left(y_{n_{i}}\right) \rightarrow h(p)$. Since $h\left(y_{n_{i}}\right) \geq \epsilon$ for all $i$, we have $h(p) \geq \epsilon$. Thus $p \notin A$. Suppose that $f^{N}(x) \subset A$ for some $N$. Then

$$
f^{N+1}(x) \subset f(A)=A, f^{N+2}(x) \subset f(A)=A, \ldots
$$

Since $n_{i} \rightarrow \infty$, we can assume that $n_{i} \geq N$. Then $y_{n_{i}} \in f^{n_{i}}(x) \subset A$. Thus $p \in \bar{A}=A$, which is a contradiction. Hence $f^{n}(x) \not \subset A$ for all $n$ and $p \in \omega(x)$.

Assume that $\omega(x) \cap A=\emptyset$. Since $\omega(x)$ is compact, there is $y \in \omega(x)$ such that $h(y)=\min h(\omega(x))$. Since $\omega(x)$ is invariant, $f(y) \subset \omega(x)$. Let $p \in f(y) \subset \omega(x)$. Since $y \notin A$, we have $h(y) \leq h(p)<h(y)$. This is a contradiction. Thus $\omega(x) \cap A \neq \emptyset$. Let $z \in \omega(x) \cap A$. There are natural numbers $m_{1}<m_{2}<\cdots$ and $z_{m_{i}} \in f^{m_{i}}(x)$ for all $i$ such that $z_{m_{i}} \rightarrow z$. Thus $h\left(z_{m_{i}}\right) \rightarrow h(z)=0$. We can assume that $m_{i}<n_{i}$ for all $i$ without loss of generality. Since $x \notin A$, $y_{n_{i}} \in f^{n_{i}}(x)$ and $z_{m_{i}} \in f^{m_{i}}(x)$, we have

$$
\epsilon \leq h\left(y_{n_{i}}\right)<h\left(z_{m_{i}}\right) \rightarrow 0 \text {. }
$$


This is a contradiction. Thus $h\left(y_{n}\right) \rightarrow 0$.

Since $f^{n}(x)$ is compact, there is $z_{x}^{n} \in f^{n}(x)$ such that

$$
h\left(z_{x}^{n}\right)=\max h\left(f^{n}(x)\right) .
$$

Since $h\left(z_{x}^{n}\right) \rightarrow 0$ as $n \rightarrow \infty$, for every $\delta>0$, there is a natural number $N(x, \delta)$ such that

$$
n \geq N(x, \delta) \text { implies } h\left(z_{x}^{n}\right)<\delta .
$$

There is a neighborhood $V_{x}$ of $x$ such that

$$
y \in V_{x} \text { implies } \max h\left(f^{N(x, \delta)}(y)\right)<\delta .
$$

Here $\max h\left(f^{N(x, \delta)}(y)\right)=h\left(z_{y}^{N(x, \delta)}\right)$.

If $n \geq N(x, \delta)$, then $h\left(z_{y}^{n}\right) \leq h\left(z_{y}^{N(x, \delta)}\right)<\delta .\left\{V_{x} \mid x \in K_{\epsilon}\right\}$ is an open cover of $K_{\epsilon}$. Since $K_{\epsilon}$ is compact, there are finitely many $x_{1}, x_{2}, \ldots, x_{k} \in K_{\epsilon}$ such that $K_{\epsilon} \subset \cup_{i=1}^{k} V_{x_{i}}$. Let $N=\max N\left(x_{i}, \delta\right)$. For any $y \in K_{\epsilon}$, there is $i$ such that $y \in V_{x_{i}}$. If $n \geq N \geq N\left(x_{i}, \delta\right)$, then $h\left(z_{y}^{n}\right)=\max h\left(f^{n}(y)\right)<\delta$. Since

$$
f^{n}(y) \subset f^{n}\left(K_{\epsilon}\right) \subset K_{\epsilon} \subset K
$$

we have $f^{n}(y) \subset K_{\delta}$ for all $n \geq N$. Thus $f^{n}\left(K_{\epsilon}\right) \subset K_{\delta}$ for all $n \geq N$.

Since $K_{\epsilon} \supset f\left(K_{\epsilon}\right) \supset f^{2}\left(K_{\epsilon}\right) \supset \cdots$,

$$
\cap_{n=0}^{\infty} f^{n}\left(K_{\epsilon}\right) \subset f^{N}\left(K_{\epsilon}\right) \subset K_{\delta} \text { for all } \delta>0 .
$$

Since $K_{\delta} \rightarrow A$ as $\delta \rightarrow 0$, we have

$$
\cap_{n=0}^{\infty} f^{n}\left(K_{\epsilon}\right) \subset A .
$$

Hence $\cap_{n=0}^{\infty} \overline{f^{n}\left(K_{\epsilon}\right)}=A$.

The proof of the theorem is complete.

\section{References}

[1] E. Akin, The General Topology of Dynamical Systems, Graduate Studies in Mathematics, 1. American Mathematical Society, Providence, RI, 1993.

[2] N. P. Bhatia and G. P. Szegö, Stability Theory of Dynamical Systems, Springer-Verlag, Berlin and New York, 1970.

[3] R. McGehee, Attractors for closed relations on compact Hausdorff spaces, Indiana Univ. Math. J. 41 (1992), no. 4, 1165-1209.

[4] M. Shub, Global Stability of Dynamical Systems, Springer-Verlag, Berlin and New York, 1987.

Gui SEOK Kim

Department of Mathematics

Hoseo University

Chungnam 337-850, Korea

E-mail address: kgs_1119@hanmail.net 
KyUnG BOK LEE

Department of Mathematics

Hoseo University

Chungnam 337-850, Korea

E-mail address: kblee@hoseo.edu

Jong SuH PARK

Department of Mathematics

Chungnam National University

TAEJON 305-764, KoreA

E-mail address: jpark@cnu.ac.kr 